\title{
Philosophical and psychological considerations when choosing educational development for students to adjust to studies
}

\author{
Nadezhda Altynova ${ }^{1^{*}}$, Andrey Orlov ${ }^{2}$, and Valentina Talantseva ${ }^{1}$ \\ ${ }^{1}$ Chuvash State Agrarian University, 428003, Cheboksary, Russia \\ ${ }^{2}$ Chuvash State University named after I. N. Ulyanov, 428015, Cheboksary, Russia
}

\begin{abstract}
According to earlier research, majority of first-year undergraduates have some difficulties adapting to the study pattern in universities. A series of papers state that this process is greatly affected by the regional realities where they live, therefore the researchers designed and tested a number of educational developments leading to better adapting. Herewith, the analysis of findings does not suggest that a combination of educational developments identified by theory and verified experimentally could be described as necessary and sufficient. It necessitates further research in this area and it's efficiency will depend on choosing the right methodology for the research. Based on the four-level methodology of scientific analysis by I.V. Blauberg and E.G. Yudin there is rationale to incorporate the provisions of the activity theory when analyzing a package of educational developments. It's implementation in physical education would ensure first-year undergraduates adapt to the study pattern in a university as effectively as possible. The main methods of research included the analysis and consolidation of scientific and methodological data, summative experiment to determine the functional state of students and correlation of the findings with their biological age. Experimentation was carried out by the Department of Physical Education, Chuvash State Agricultural Academy, Russia. The research resulted in supplementing the earlier proposed set of educational developments with other two. The first of them is to objectify the need for physical exercise as an effective way to enhance adaptation of firstyear students. The second one is to have monitoring and corrective measures in place as part of the activity. Together with the earlier recommended framework, these educational developments ensure all functionalities of the activity to improve the studied process of first-year students adaptation to the study pattern in a university. Whereby a set of educational developments could be termed as a coherent organic system
\end{abstract}

\section{Introduction}

A retrospective analysis of psychological and pedagogical literature shows that the process of first-year students adaptation to the study pattern in a university is somewhat lengthy $[1,2]$. It is evident by the fact that they feel out of place and find it difficult to interact with each other

\footnotetext{
*Corresponding author: naltynova_777@mail.ru
} 
in a new environment. Under certain circumstances, this may lead to a nervous breakdown and, thus, cause low academic performance in the first semester.

Researchers competent in this problem believe that students adapt to studies at a university differently, for some students it is smooth, for others it leapfrogs. This could be due to both personal traits of character, different type of higher nervous activity, and a combination of social factors $[3,4,5,6]$. In this regard, in order to shorten the period of adjusting to the training curriculum, specific forms of engagement with new students are designed on-site, implemented at:

- $\quad$ educational level (adjusting to the studies);

- psychological level (promoting motivation for activities aimed at successful adaptation);

- $\quad$ interpersonal level (ensuring effective interpersonal interaction processes) [7, 8].

However, successful adjustment of students to the study pattern at a university largely depends on the functional state of a student. Hence, we believe that having certain educational developments in place will allow first-year students to better adjust to the studies in higher school, which defined the objective of our study.

\section{Materials and methods}

Theoretical and methodological basis of the research became the four-level methodology of scientific analysis of I. V. Blauberg and E. G. Yudin [9], the provisions: materialist dialectic of contradiction as a force behind development; the activity theory to objectify the needs and functionalities of activity.

The main research methods were analysis and aggregation of data from psychological and pedagogical literature, summative assessment, mathematical processing of experimental data and their comparison using Voytenko's method.

Examinees' ability to keep balance was tested using Romberg's test: keeping balance with closed eyes while standing on one leg (the heel of the other leg touches the ball of the knee) with arms extended forward with open fingers. Holding time was measured in seconds until shaky hands and body position change.

\section{Results and discussion}

Under the chosen research methods that involve coherent reflection on the studied problem at philosophical, general scientific (systemic), specifically scientific and technological levels, we may conclude that most studies of first-year students adjustment to the study pattern in a university are usually limited to a specifically scientific level of learning. Hence, the findings from the scientific community gained during experiments are individual, thereby making them more difficult to introduce in other educational institutions. Nevertheless, the foregoing does not compromise the value of earlier studies.

Among many researches on this issue, of particular interest are the works that identify educational developments to improve adjustment of first-year students to the study pattern in a university taking into account regional realities where they live [10]. Using the questionnaire proposed by Rogers and Diamond, the authors draw out personalities of first-year students of intramural and extramural forms of studies, and the level of their socio-psychological adaptation. $86 \%$ of intramural students were found to have very low adaptation parameters, thus feeling rather uncomfortable in a new educational environment, struggling in subject mastery. $10 \%$ of examinees showed satisfactory adaptation, and only $4 \%$ had high level of adaptation allowing them to rather easily access to curricula and be proactive in other activities (cultural, sports, research events, etc.). 
Extramural students showed totally different trend. Only $20 \%$ of examinees were poorly adaptive, $48 \%$ had satisfactory level, and $32 \%$ of respondents had regular and high level of adaptation.

Mental stability assessment revealed that $68 \%$ of intramural first-year students were more likely to have nervous breakdowns even in small stressful situations (tests, answering near blackboard, lab work presentation, etc.). An average mental to stress tolerance had $28 \%$ of firstyear students, $4 \%$ were highly stress tolerant, respectively. Herewith, only $12 \%$ of extramural students had low mental to stress tolerance, $53 \%$ of examinees had average level, and $35 \%$ were highly tolerant to stress.

The findings let the authors to conclude that extramural students whose majority were middle-aged people were better adaptive and mentally stable. While the vast majority just-outof-school students were poorly adjustive and were more likely to have nervous breakdowns [10].

It is common knowledge that the adaptation is when the organism adjusts to environmental irritating factors (stressors). For students starting their first year at an institution of higher education, adaptation means adjusting to stressors such as change of daily routine and diet, relocation of residence, more dynamic learning process, etc.

If we look at adaptation from a physiological point of view, it should be noted that coping with a stressful situation is mainly about the release of thyroid hormones. They provide energy together with adrenal gluco- and mineralocorticoids. Thyroid hormones deficiency is known to impair the differentiation of cortical neurons. They among other things (metabolic slowdown, physical weakness, weak immune system leading to diseases becoming chronic, weak reproductive function) affect person's mental abilities.

Noting the importance of thyroid hormones in regulating metabolic processes and people's activities in general, it should be noted that the Chuvash Republic, Russia is a goiter-endemic area due to low iodine content in soil and water. Thus, local population has mild to moderate iodine deficiency and is more vulnerable to endocrine disorders. Over the last years, Chuvashia has no routine prevention of endemic goiter, only rare families supplement their diet with iodine [10]. All this prompted the authors to conduct a research. It revealed that first-year students with endemic goiter find it harder to adjust to a new learning environment. Drawing on the knowledge gained, a set of educational developments (diagnostic assessment of student adaptability parameters; Physical Education and Sports discipline was supplemented with the course "Endemic goiter - regional pathology, the influence of thyroid hormones on physical and mental performance of a person"; applying individual and differentiated approach in the learning process) were introduced and successfully tested improving first-year students' adjustment to the study pattern in higher school.

At the same time, as the authors of the study claim, a comparative analysis of the examinees outcome showed little difference between the parameters of the control and experimental groups. We believe the findings serve the basis to search for further educational developments that would catalyze first-year students' adjustment to the study pattern at a university.

From the philosophy course we know that the main source of an object or phenomenon development, regardless of the nature of its origin, is a contradiction. Extrapolating this into practice of the studied problem helps to identify what could become stimulus for first-year students to engage in physical activities as a key to improving their adaptive capacity. This is needed since, according to statistics, the number of people engaged in regular physical activity of different forms does not exceed $15 \%$ of university students [11], attendance of PE classes is mainly motivated by getting a final pass $[12,13,14]$. Perhaps this is because most students have their need for physical health subjectively satisfied. It doesn't motivate them to take regular physical exercise. Using the concepts of the activity theory and following the "mechanism for objectifying a need" which results in a motive for the activity being formed (A.N. Leontev, L.S. Vygotskiy, S.L. Rubinstein, Yu. B. Gippenreiter, etc.), this need must be translated from the 
potential state to the current one. In this respect, it is interesting to note that many students are highly concerned about how their functional state correlates against their biological age. Using the Romberg's test, we assessed the ability of first-year students to balance which is one of the measures of wellness [15]. The findings were interpreted in accordance with the biological age score using Voytenko's method [16]. We chose this test since, firstly, it rather shows the state of the nervous system and vestibular apparatus than the level of basic physical skills. Secondly, it does not require sophisticated instrument. It is quick to do and easy to calculate the results.

The experiment deliveries in Figure 1 clearly show that the vast majority of examinees have a biological age of 65 years or above.

These results propelled the students to rethink their attitude to their health and thus making their need for physical exercise mainstream. This leads us to a conclusion that interpreting these functional tests against biological age may serve an additional educational development to improve first-year students' adjustment to the studies at a university. We also believe this development will be primary relative to the other three identified during earlier researches [10].

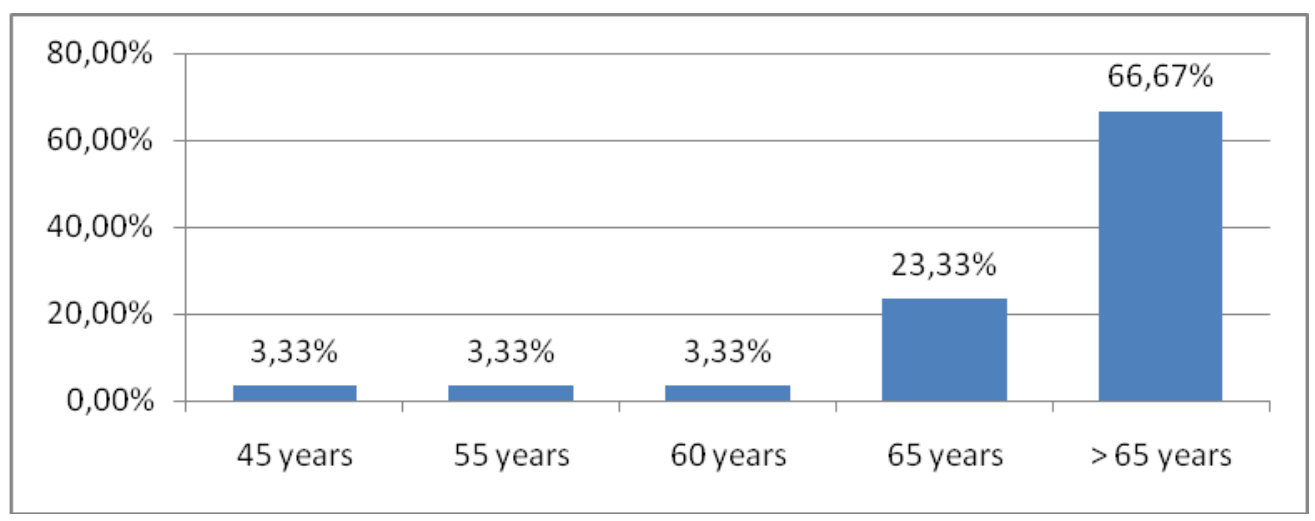

Fig. 1. Results of the Romberg's test in students of Chuvash State Agricultural Academy compared against a biological age score according to Voytenko's method

In addition to the above and looking at the process of first-year students' adjustment to the research pattern at a university as a specific activity, it should be taken into account that activity includes such functionalities as a motive for the activity (M), orienting basis of activity (OBA), executive part of activity (EPA) and monitoring and corrective part of activity (MCPA) (according to A.N. Leontev, S.L. Rubinstein, L.S. Vygotskiy). If the motive to act to adapt to the research pattern at a university is formed easily when first-year students see their biological age test result, the necessary theoretical knowledge as orienting basis for adaptation activities is provided by a PE teacher. The executive part of activity is carried out by the students themselves. And only to ensure this activity is effective, and to have higher chances for a desired result, a system of corrective actions needs to be developed. It would be mandatory if the achieved result is not as planned. The reason behind this is that monitoring of activities is in place by default (Yu. B. Gippenreiter). While correction is only when necessary. The above allows considering the designed system of corrective measures as the fifth development for structuring the adaptation process for first-year students.

\section{Conclusions}

Theoretical analysis of the first-year students adjustment of structuring process problem to the study pattern at a university and its interpretation under four-level methodology of scientific 
analysis shows that earlier highlighted educational developments to catalyze the process under study will be added with other two that we formulated taking into account the activity theory provisions. In this case, the proposed educational developments can be described as necessary and sufficient to ensure effective structuring of the studied process. Besides, they are considered as systemic interaction where the first development which is subordinate to others stipulates their functioning. Further three actually serve to form the orienting basis and ensure executive part of students' activities to boost their adaptive capacity. The fifth development ensures that the subject of activity (a first-year student) is able to correct it when the planned result is not achieved. If we take into account the fact that, under the activity theory, there is no clear distinction between the orienting basis, its executive, and monitoring and corrective parts (they represent the unity), we may claim that, unlike the first mentioned educational development, the said ones are in coordination interaction with each other. Herewith, this set of developments forms a certain integral and organic system. It presupposes a synergistic effect when implemented since it involves formation and development of all functional parts of activity (M, OBA, EPA, MCPA) of first-year students to boost their adaptive capacity.

This thesis of educational developments system advanced as a scientific hypothesis needs further research and additional educational experiment to test them.

\section{References}

1. A.M. Balabanova, Young scientist, 14, 254-256 (2018)

2. L. S. Elgina, Bulletin of the BSU. Education. Personality. Society, 5 (2010).

3. L.A. Antipova, Kazan Pedagogical Journal, 2, $52-56$ (2008)

4. V.N. Bolgov, Z.M. Kuznetsova, Dialogues of the New Century Conference Proceedings, 346-347 (2003)

5. N.A. Grankin, Z.M. Kuznetsova, Pedagogico-psychological and medico-biological problems of physical culture and sports, 12(3), 37-46 (2017)

6. A.I. Pyanzin, Science and sports: current trends, 1(2), 33-45 (2014)

7. M. Gerber, S. Ludyga, M. Mucke, F. Colledge, S. Brand, U. Puhse, Psychoneuroendocrinology (2017)

8. T. Degtiarenko, R. Yagotin, Nauka i osvita (2017)

9. I.V. Blauberg, Yudina E.G. Formation and essence of the system approach (Science, Moscow, 1973)

10. N.V. Altynova, Problems of the contemporary teacher education, 59-3, 16-21 (2018)

11. A.I. Orlov, A.G. Shugaev, A.N. Ivanov, Proceedings of International Practical Conference, Cheboksry-Tashkent, 147-151 (2018)

12. L.S. Elgina, Bulletin of the BSU. Education. Personality. Society, 5 (2010)

13. V.A. Pitkin, D.D. Ivanova, L.A. Kholodnaya, Problems of modern education, 5 (2019)

14. M.G. Shnayder, N.N. Pyanzina, O.B. Kolesnikova, Actual problems of physical culture and sports in modern socio-economic conditions: Materials of the International Scientific and Practical Conference dedicated to the 100th anniversary of the National University of Uzbekistan named after Mirzo Ulugbek, May 17-18, 2018 (NUU named after Mirzo Ulugbek, Cheboksary: Chuvash State Agricultural Academy, Tashkent, 175-181, 2018)

15. Do You Know Your Age

URL:https://zen.yandex.ru/media/id/5ad254f20422b4c791a59b35/a-vy-znaete-svoi-biologicheskiivozrast-test-5b8ab5833c9a2400a9a120be

16. Collection of biological age tests. URL https://beautyaura.ru/tests/bioage/biological-age-test.html 